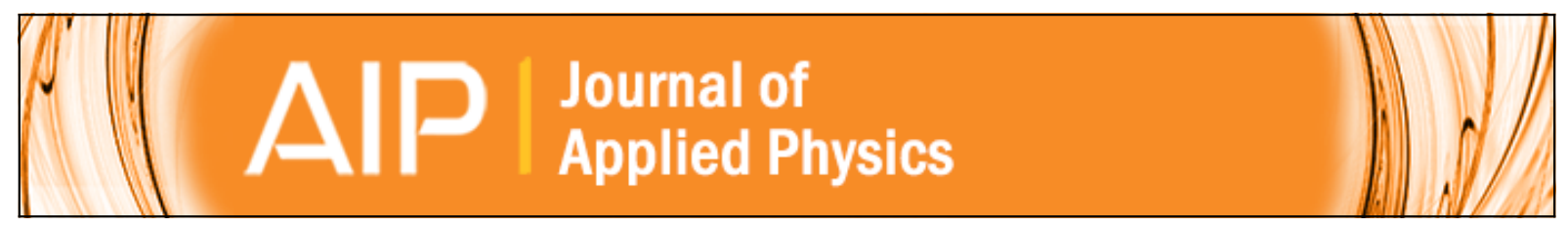

\title{
Subpicosecond laser ablation of dental enamel
}

A. V. Rode, E. G. Gamaly, B. Luther-Davies, B. T. Taylor, J. Dawes, A. Chan, R. M. Lowe, and P. Hannaford

Citation: Journal of Applied Physics 92, 2153 (2002); doi: 10.1063/1.1495896

View online: http://dx.doi.org/10.1063/1.1495896

View Table of Contents: http://scitation.aip.org/content/aip/journal/jap/92/4?ver=pdfcov

Published by the AIP Publishing

\section{Articles you may be interested in}

Evaluation of efficiency of laser systems on removal of debris and smear layer in maxillary canine root canals J. Laser Appl. 26, 032005 (2014); 10.2351/1.4870878

Assessment of external root temperature during root canal irradiation by Nd:YAG and Er:YAG lasers

J. Laser Appl. 21, 119 (2009); 10.2351/1.3184428

A mathematical description of surface texture development in laser-ablated dentin

J. Appl. Phys. 100, 104701 (2006); 10.1063/1.2369633

Laser ablation of human tooth

J. Appl. Phys. 97, 094919 (2005); 10.1063/1.1897494

Cementum melting after dye-assisted holmium laser irradiation

J. Laser Appl. 16, 193 (2004); 10.2351/1.1771339

\section{AIP $\left.\right|_{\text {APL Photonics }}$}

APL Photonics is pleased to announce Benjamin Eggleton as its Editor-in-Chief

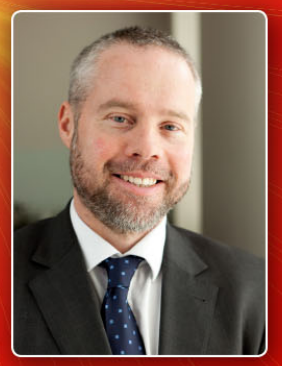




\section{Subpicosecond laser ablation of dental enamel}

A. V. Rode, ${ }^{\text {a) }}$ E. G. Gamaly, and B. Luther-Davies

Laser Physics Center, Research School of Physical Science and Engineering, Australian National

University, Canberra, ACT 0200, Australia

B. T. Taylor and J. Dawes

Centre for Lasers and Applications, Department of Physics, Macquarie University, New South Wales 2109, Australia

A. Chan ${ }^{\text {b) }}$

Caringbah, New South Wales 2229, Australia

R. M. Lowe and P. Hannaford

Centre for Atom Optics and Ultrafast Spectroscopy, Swinburne University of Technology, Hawthorn, Victoria 3122, Australia

(Received 27 December 2001; accepted for publication 31 May 2002)

Laser ablation of dental enamel with subpicosecond laser pulses has been studied over the intensity range of $(0.1-1.4) \times 10^{14} \mathrm{~W} / \mathrm{cm}^{2}$ using 95 and $150 \mathrm{fs}$ pulses at a pulse repetition rate of $1 \mathrm{kHz}$. The experimentally determined ablation threshold of $2.2 \pm 0.1 \mathrm{~J} / \mathrm{cm}^{2}$ was in good agreement with theoretical predictions based on an electrostatic ablation model. The ablation rate increased linearly with the laser fluence for up to 15 times the ablation threshold. The absence of collateral damage was observed using optical and scanning electron microscopy. Pulpal temperature measurements showed an increase of about $10^{\circ} \mathrm{C}$ during the $200 \mathrm{~s}$ course of ablation. However, air cooling at a rate of $5 \mathrm{l} / \mathrm{min}$ resulted in the intrapulpal temperature being maintained below the pulpal damage threshhold of $5.5^{\circ} \mathrm{C}$. The material removal rates for subpicosecond precision laser ablation of dental enamel are compared with other techniques. (C) 2002 American Institute of Physics.

[DOI: $10.1063 / 1.1495896$ ]

\section{INTRODUCTION}

Since the first use of lasers in the medical field, contactfree application of laser light for removal of hard dental tissue has been studied as a means for replacing conventional surgical tools. To date, lasers have not succeeded in replacing the dental drill in many hard tissue applications due to slow material removal rates and unacceptable collateral damage. For conventional pulsed laser ablation with pulses from 100 ps to microseconds, a strong thermal shock wave is accompanied by cracking of the remaining bulk material and inefficient, uncontrolled material removal. ${ }^{1}$ However, in recent years the development of high-average-power, highrepetition-rate subpicosecond lasers is causing a rebirth of interest in laser surgical applications due to their precise and highly effective ablation capabilities and minimal thermal and shock wave collateral damage. ${ }^{1-8}$

The shock wave, vibration, and rise in temperature in bulk dental tissue surrounding the spot treated can cause significant pain to the patient. These effects can be eliminated with a decrease of the laser pulse duration to the subpicosecond time regime and a consequent increase of the peak laser intensity. Let us highlight the essential features of material ablation by powerful subpicosecond laser pulses.

The subpicosecond laser pulse duration is shorter than the characteristic energy relaxation times, i.e., the electron-

\footnotetext{
${ }^{a)}$ Electronic mail: avr111@ rsphy1.anu.edu.au

${ }^{b)}$ Private dental practitioner.
}

to-ion energy transfer time and the heat conduction time. Thus, no hydrodynamic (ion) motion can occur during such a short laser pulse, and conventional thermal ablation mechanism is prohibited. However, if the laser intensity exceeds $10^{13}-10^{14} \mathrm{~W} / \mathrm{cm}^{2}$ the collisional, or bremsstrahlung, mechanism of laser light absorption in the ionized atoms on the target surface becomes very efficient, since it is of the same nature as that in metals or in plasma. The laser pulse can ionize the target material early on in the laser pulse at this intensity. If the laser energy absorbed by the electron exceeds the sum of the first ionization potential and the energy required to leave the solid target (the work function in metals), then the electron can escape the target, carrying away a substantial amount of energy. The escaped energetic electron creates a huge electric field due to charge separation with the parent ion. This process of ablation via the electrostatic field of charge separation, to which we refer further as "electrostatic ablation," occurs during the subpicosecond pulse duration.

For real surgical and industrial applications it is crucial to choose the correct laser parameters-wavelength, pulse duration, and intensity - in order to ablate a particular material. Taking into account the complexity of the physical processes involved and the complex chemical composition of dental enamel, the problem is difficult, if not impossible, to solve from first principles. We have recently developed simple analytical scaling laws for the ablation thresholds of metals and dielectrics under irradiation with powerful subpicosecond laser pulses. ${ }^{9,10}$ The physics of this new ablation 
regime involve ion acceleration in the electrostatic field caused by the charge separation created when the energetic electrons escape from the target. The formulas derived from the theoretical model for the ablation thresholds and the ablation rates for metals and dielectrics ${ }^{9}$ link together the laser and target parameters. The calculations appear consistent with the experimental data in the femtosecond range. The calculated ablation thresholds extrapolate continuously to the thermal ablation regime for longer picosecond and nanosecond laser pulses.

In this article we present experimental data on laser ablation of dental enamel in the ultrashort laser-matter interaction regime. The ablation threshold and the ablation rate were measured as a function of the laser fluence. Simultaneous measurements of the intrapulpal temperature rise in the tooth were conducted during ablation. The laser parameters for effective ablation of the enamel were derived from our analytical model. ${ }^{9}$ An analysis of the experimental data allowed the average binding energy to be determined for the complex composition of dental enamel. We also analyzed the crater floor and characterized the collateral damage in the bulk of the ablated teeth with the aid of optical and electron microscopy. So we present recommendations for precise laser machining of dental enamel by subpicosecond laser pulses.

\section{EXPERIMENTS}

Two separate Ti:sapphire laser systems were used in the experiments. The first was a $780 \mathrm{~nm} 150$ fs Clark-MXR CPA-2001 laser system at the Australian National University with an average laser power of $0.5-0.6 \mathrm{~W}$. The second, a SpectraPhysics Tsunami laser and Spitfire regenerative amplifier at Swinburne University of Technology generated 95 fs pulses at $805 \mathrm{~nm}$ and average powers of $0.8-1.0 \mathrm{~W}$. Both lasers operated at a $1 \mathrm{kHz}$ pulse repetition rate. The energy stability in both lasers was typically within $2 \%$, thereby providing high-quality beam intensity profiles for uniform interaction on the target surface. However, there was substantial scatter in the ablation rate measurements, up to $\pm 50 \%$ at fixed laser power, which we attribute to the roughness of the enamel surface and inhomogeneity in the bulk of different teeth. The laser pulses were focused onto the tooth surface using a $250 \mathrm{~mm}$ focal length scanning lens to a focal spot of $\sim 50 \mu \mathrm{m}$ full width at half maximum (FWHM) (focal spot area $2.2 \times 10^{-5} \mathrm{~cm}^{-2}$ ), providing a maximum laser intensity of around $1.4 \times 10^{14} \mathrm{~W} / \mathrm{cm}^{2}$ with 150 fs pulses and 3.8 $\times 10^{14} \mathrm{~W} / \mathrm{cm}^{2}$ with $95 \mathrm{fs}$ pulses (maximum laser fluences of 21 and $36.5 \mathrm{~J} / \mathrm{cm}^{2}$, respectively). The laser intensity was varied by inserting neutral density filters into the beam. In one experiment the $95 \mathrm{fs}$ pulses were converted to the second harmonic to determine the difference in ablation threshold for 800 and $400 \mathrm{~nm}$ radiation.

An important condition for ultrashort pulse interaction mode is the high contrast ratio of the pulse: the target surface should not be ionized, damaged, or ablated during pre-pulse action of the amplified spontaneous emission. For the nanosecond-scale pre-pulse and the 100 fs main pulse the energy contrast ratio was measured in both laser systems to be of the order of $>10^{4}$.

The laser beam was scanned over a $1 \times 1$ or a $2 \times 2 \mathrm{~mm}^{2}$ tooth surface area with a dual-axis galvanometer beam scanning system and formed a square crater. This allowed easy and precise evaluation of the ablated volume, which was determined by measuring the crater depth after a period of $100-600 \mathrm{~s}\left(1-6 \times 10^{5}\right.$ laser pulses). The mirrors' scanning speed was rather slow, about $50 \mathrm{~Hz}$, in order to prevent the formation of deep pockets at the crater edges due to the inertia of the scanning mirrors at the turning points. However, it was high enough to guarantee a single laser shot per site and to avoid overlapping of two consecutive pulses on the tooth surface.

The crater depth was defined using a calibrated microscrew by focusing a microscope objective to the top of the tooth surface and subsequently to the floor of the ablated crater. The average uncertainty in the measurements of the ablated depth per pulse was of the order of $0.1 \mu \mathrm{m}$. The crater depth measurements were verified with nanoprofilometer scans and with electron micrographs of the walls of the crater. The ablated depth per pulse was determined by dividing the ablated volume on the product of the number of pulses and the focal spot area. The ablation threshold values were extrapolated from experimental data of the ablated depth versus the laser fluence.

Ablation rate studies were performed on healthy enamel of human incisors and molars. The teeth were treated with $10 \%$ sodium hydroxychloride for $10 \mathrm{~min}$ and then stored in formalin. Teeth used for pulp cavity temperature measurements were sliced longitudinally in half with the pulp scraped out. Some sliced teeth halves were clamped together and the ablation area was extended over the cut. This allowed postexamination of the layer affected under the crater floor. Pulpal temperature measurements were taken with a thermocouple attached to a digital thermometer. The thermocouple was placed in the pulpal chamber of the teeth and secured using superglue. The area was then sealed using epoxy resin. The temperature measurements were accurate to $0.1^{\circ} \mathrm{C}$, and were taken every $0.2 \mathrm{~s}$.

To compare the crater features of short-pulse and longpulse ablation, we performed a few ablations using a $Q$-switched mode-locked Nd:YAG laser. The $60 \mathrm{ps}$ laser pulses at $1.064 \mathrm{~nm}$ followed in trains of $\sim 220-250 \mathrm{~ns}$ duration $(\sim 17$ pulses per train) with a train repetition rate of 500 $\mathrm{Hz}-2 \mathrm{kHz}\left(1-4 \times 10^{4}\right.$ pulses per second); the average laser power was $\sim 2.7-7.9 \mathrm{~W}$, respectively $(200-300 \mu \mathrm{J} /$ pulse $)$. The laser was focused down to a $25 \mu \mathrm{m}$ spot [laser intensity of $\sim(7-10) \times 10^{11} \mathrm{~W} / \mathrm{cm}^{2}$, fluence of $\left.40-60 \mathrm{~J} / \mathrm{cm}^{2}\right]$, and scanned over the same size area on the tooth surface. The laser repetition rate was chosen so as to maintain the rise in intrapulpal temperature during the $10 \mathrm{~s}$ ablation below $4{ }^{\circ} \mathrm{C}$.

\section{EXPERIMENTAL RESULTS}

\section{A. Optical and scanning electron microscope images}

The ablated areas were examined with an optical microscope and a scanning electron microscope (SEM). The images of the craters ablated with subpicosecond lasers show 


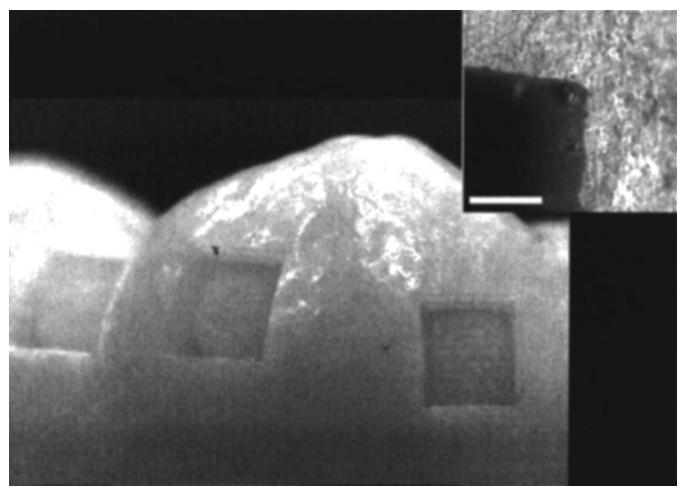

FIG. 1. Optical microscope images of the $1 \mathrm{~mm}^{2} \times 0.25 \mathrm{~mm}$ craters on the tooth surface ablated with 150 fs laser pulses. The image of the crater's cut edge in the inset shows the absence of any collateral damage in the surrounding tissue. The scale bar is $50 \mu \mathrm{m}$.

the absence of any collateral damage at the crater edge (Fig. 1). This observation was supported by the SEM images (Fig. 2).

It can be seen from Figs. 2(b), 2(d), and 2(f) that the enamel ablated with 60 ps pulses showed clear evidence of thermal damage to the tooth. Each ablated square had a darkened ring approximately $0.3 \mathrm{~mm}$ from the edge of the square, as well as enamel droplets on the surface of the tooth next to the squares. The enamel surface near the edge of the crater
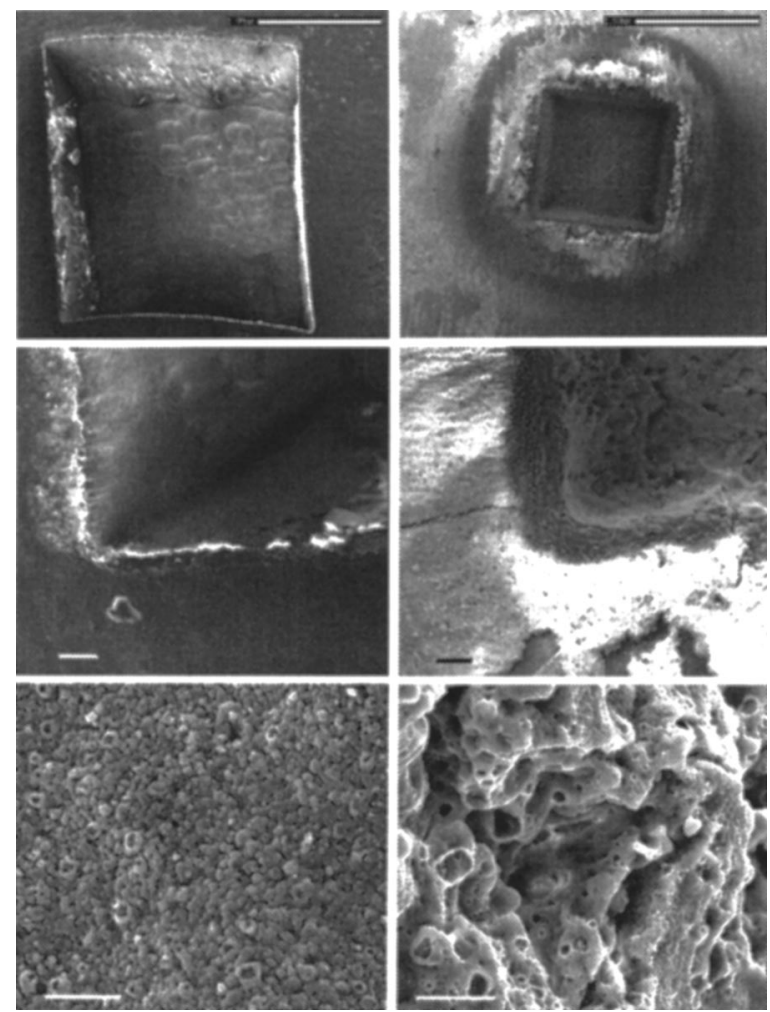

FIG. 2. SEM images of $0.3 \mathrm{~mm}$ deep craters in enamel ablated with $150 \mathrm{fs}$ laser (left) and 60 ps laser pulses (right). There is no collateral damage around the fs laser ablated cavity; the edges are sharp and the crater floor is smooth. With 60 ps laser there is a thermally modified area around the crater and some occasional cracks. The scale bars are $1 \mathrm{~mm}$ in the top two images and $10 \mu \mathrm{m}$ in the others.

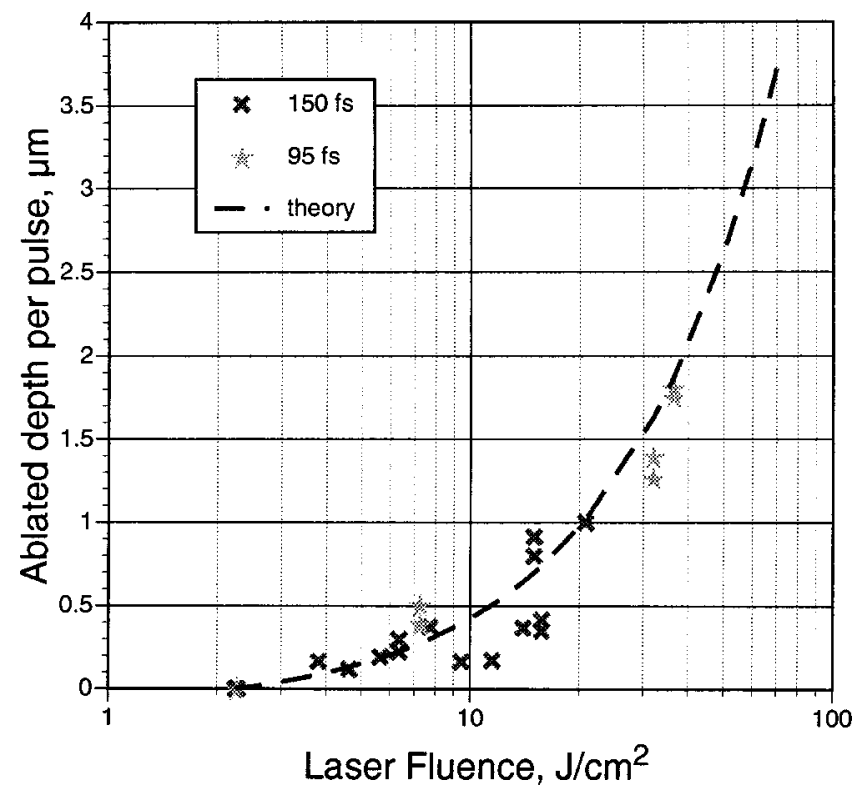

FIG. 3. Ablated depth vs laser fluence with 95 (stars) and 150 fs (crosses) pulses. The line is the theoretical prediction based on Eq. (4), taking the experimentally determined ablation threshold of $2.2 \mathrm{~J} / \mathrm{cm}^{2}$ for both pulse durations.

was raised due to heat deposition in the bulk of the tooth. The floor of the ablated craters appeared melted and uneven.

In sharp contrast to the picosecond pulses, the enamel ablated by 95 and 150 fs pulses [seen in Figs. 2(a), 2(c), and $2(\mathrm{e})]$ showed no collateral thermal damage at all. The walls and floor of these ablated squares were also smoother than those due to picosecond ablation and the edges and corners were sharper than those ablated with 60 ps pulses.

\section{B. Ablation rate measurements}

The measured ablation rates over the intensity range of $10^{13}-4 \times 10^{14} \mathrm{~W} / \mathrm{cm}^{2}$ with both 95 and $150 \mathrm{fs}$ pulses are summarized in Fig. 3. The ablation threshold fluence $F_{\text {th }}$ was found to be $2.2 \pm 0.1 \mathrm{~J} / \mathrm{cm}^{2}$. The threshold was about the same for 95 and for 150 fs pulses. An optical microscope test confirmed that there was no visible damage to the enamel surface at the laser fluence below this threshold value. The ablation threshold with $400 \mathrm{~nm}$ (second harmonic of $95 \mathrm{fs}$ pulse) was found to be $1.0 \pm 0.2 \mathrm{~J} / \mathrm{cm}^{2}$.

The ablation rates increased linearly above the threshold level for the available laser fluences up to $36.5 \mathrm{~J} / \mathrm{cm}^{2}$, i.e., up to $\sim 15 F_{\text {th }}$. There was no detectable difference, within the accuracy of measurements, in the ablation rate with 95 and 150 fs laser pulses. The most efficient ablation was observed at the maximum laser fluence (intensity) of $36.5 \mathrm{~J} / \mathrm{cm}^{2}$ (4 $\times 10^{14} \mathrm{~W} / \mathrm{cm}^{2}$ ), which yielded $1.8 \mu \mathrm{m}$ of ablated depth per 1 $\mathrm{mJ}$ pulse. This value is in reasonable agreement with the results ${ }^{1}$ published for $350 \mathrm{fs} 1.053 \mu \mathrm{m}$ pulses at a pulse repetition rate of $10 \mathrm{~Hz}$.

The threshold fluence extrapolated from the ablation rate measurements for dental enamel is about three times higher than that measured in Ref. 11. The difference could be due to the following reasons. First, 100 pulses were hitting the same spot on the enamel surface, leading to accumulation of the 


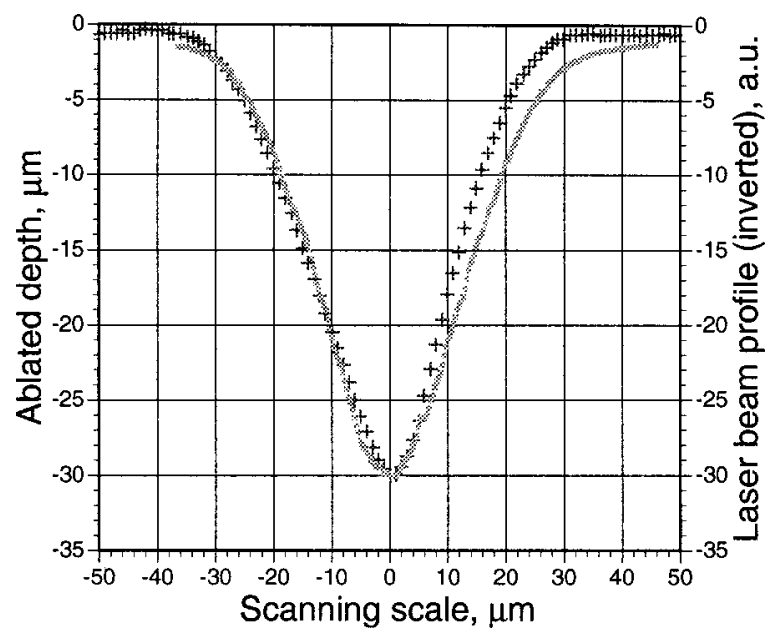

FIG. 4. Spatial distribution of the crater profile (crosses) and of the inverted laser pulse profile, illustrating the linear dependence of the ablated mass on the laser intensity.

effect of surface damage in Ref. 11, while in our experiments the threshold was determined for a single pulse. The second reason could be related to the difference in enamel surfaces treated with laser pulses. In the experiments presented here the outer surface of incisors and molars might be harder than the sliced enamel surfaces of molar teeth used in the experiments in Ref. 11. The third reason could be related to the method of threshold determination. The method of extrapolation of ablated depth values formed by single pulses of various laser fluences to the "zero" depth, used in this article, provides a physically meaningful value for the action of the main subpicosecond pulse without any low-energy prepulse. The damage threshold detected by scanning electron microscopy in Ref. 11 may take into account the action of the amplified spontaneous emission as well as of a possible laser prepulse on the enamel surface, particularly considering the accumulated impact of 100 laser pulses.

To test the linear dependence of the ablated mass on the laser intensity, we performed the ablation at the maximum available laser fluence of $36.5 \mathrm{~J} / \mathrm{cm}^{2}$, with the laser beam scanning along one axis only over the enamel surface, and measured the cross section of the ablated channel with a nanoprofilometer. The resulting profile is presented in Fig. 4. Also in Fig. 4 we present an inverted laser beam profile, normalized to the level of maximum depth in the channel. As can be seen, the channel profile faithfully reproduces the spatial intensity distribution in the laser beam. This result confirms that at laser fluence level up to $15 F_{\text {th }}$ the ablated mass is directly dependent on the laser intensity as suggested by the rate measurements.

\section{Intrapulpal temperature measurements}

Measurements of the rise in the intrapulpal temperature are shown in Fig. 5. The curves represent the results of evaporation sessions of $200 \mathrm{~s}$ each without and with air cooling the teeth at various air flow rates. The experiments with a number of teeth show that the total increase of temperature is about $10^{\circ} \mathrm{C}$, with variations from tooth to tooth depending on the distance from the ablated area to the tip of the ther- (a)
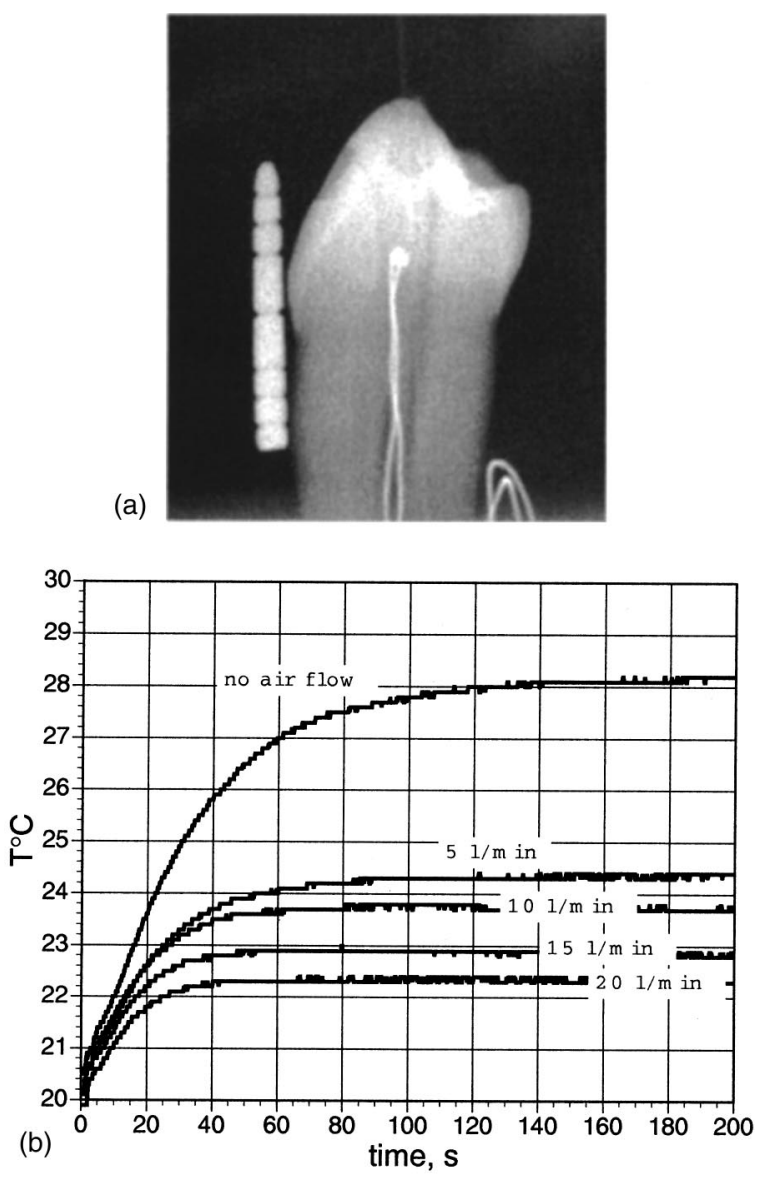

FIG. 5. X-ray image of a tooth with a thermocouple embedded (a) and the intrapulpal temperature of the ablated tooth without air cooling and with air cooling at various air flow rates (b).

mocouple placed in the pulp cavity. When the teeth were cooled by air blown from a distance of $10 \mathrm{~mm}$ at various flow rates from 5 to $20 \mathrm{l} / \mathrm{min}$ (Fig. 5), the temperature was observed to saturate at a level below the pulp thermal damage limit of $5.5^{\circ} \mathrm{C}$. Since Zach and Cohen ${ }^{12}$ showed that with a rise of intrapulpal temperature over $5.5^{\circ} \mathrm{C}, 15 \%$ of the tooth pulp may suffer irreversible thermal damage, and this value is considered critical.

\section{SCALINGS FOR THE THRESHOLD ENERGY DENSITY AND THE ABLATION RATES}

A detailed description of the electrostatic ablation mode with ultrafast laser pulses is presented elsewhere. ${ }^{9}$ Below we apply the scaling formulas derived from this model to the experimental conditions to estimate the ablation threshold and ablation rates, and finally to choose optimal laser parameters for ablation of dental enamel.

The main condition for ultrashort laser-matter interaction mode is that the pulse duration is shorter than all the relaxation times: the heat conduction time and the electronto-ion energy transfer time. In order to ablate the target during interaction mode, the electrons should absorb the energy above the ionization potential and the energy of the potential barrier required to escape from the target surface. Each of the escaped electrons creates an electric field of charge separation with the parent ion. If the energy of the escaped elec- 
trons is higher than the ion binding energy, then the ions are pulled out of the laser-affected spot on the target surface. This electric field is the physical reason for the nonthermal mechanism of ablation with subpicosecond laser pulses. ${ }^{9,13}$ The difference in the new regime of ultrafast ablation, electrostatic ablation, is based on the ion extraction by the electrostatic field of the charge separation created by the energetic electrons escaping from the target during the pulse. The mechanism of electrostatic ablation terminates after the laser pulse. For this reason, all the processes of energy transfer from the laser pulse to the bulk of the target material, which are the reason for the accompanying collateral damage, are prevented. There is no melting or evaporation in a conventional equilibrium sense in the electrostatic ablation regime.

In a case where the absorbed laser energy is lower than the threshold energy for electrostatic ablation, the electrons transfer the energy to the lattice through electron-phonon interaction, and the ablation process occurs mainly after the laser pulse in a way similar to thermal laser ablation with longer pulses. ${ }^{14,15}$ In actual practice, the spatial and temporal intensity distributions in the laser pulse result in a mixed interaction mode. However, at the laser intensity range above $10^{13} \mathrm{~W} / \mathrm{cm}^{2}$ the proposed scaling leads to the electrostatic ablation mechanism, which then dominates in the lasermatter interaction process.

For laser intensities of $10^{13}-10^{14} \mathrm{~W} / \mathrm{cm}^{2}$, ionization is completed at the beginning of the laser pulse for most target materials. The laser energy is absorbed by free electrons in a thin skin layer in a similar way for both metals and dielectrics. The time and space dependence of the electron energy in the skin layer can be expressed with in the framework of the normal skin effect as ${ }^{9}$

$$
\epsilon_{e}=\frac{2}{3} \frac{A I_{0}}{l_{s} n_{e}} \exp \left\{-\frac{x}{l_{s}}\right\}
$$

where $I_{0}$ is the laser intensity. $A$ is the absorbance and $l_{s}$ is the skin depth, both of which are functions of the laser frequency $\omega_{0}$, the number density of conductivity electrons $n_{e}$ (or plasma frequency $\omega_{p e}$ ), the electron-ion collision frequency, and the angle of incidence and polarization of the laser beam.

We introduce the electrostatic ablation threshold for dielectrics by the following condition: the energy of the electrons from the surface layer (i.e., thickness $\ll l_{s}$ ) must reach the sum of the atomic binding energy $\epsilon_{b}$ and the ionization potential $J_{i}$ by the end of the laser pulse $t_{p}$. Hence, the threshold fluence for dielectrics $F^{d}$ from Eq. (1) is as follows:

$$
F_{\mathrm{th}}^{d} \equiv I_{0} t_{p}=\frac{3}{4}\left(\epsilon_{b}+J_{i}\right) \frac{l_{s} n_{e}}{A}
$$

here $n_{e}$ is approximately equal to the atomic number density $n_{a}$, as in a single-ionized plasma. By applying the approximate relation $A / l_{s} \cong 2 \omega / c,{ }^{9}$ the threshold fluence increases in direct proportion to the laser wavelength:

$$
F_{\mathrm{th}}^{d}=\frac{3 \lambda n_{e}}{16 \pi}\left(\epsilon_{b}+J_{i}\right)
$$

The average evaporation rate, which is the number of atoms evaporated per unit area per second, was estimated using the energy conservation law for the ultrashort interaction mode in the same way as it was done for the long laser pulses, namely, the total absorbed laser energy flux in the optimum ablation mode must be balanced by the outflow of ablated atoms times the energy loss per atom. ${ }^{16,17}$ This energy per atom approximately equals the binding energy, ionization energy, and the electron temperature. Thus, the ablation rate for the ultrashort regime is given by

$$
n v\left[\mathrm{~cm}^{-2} \mathrm{~s}^{-1}\right] \cong \frac{A I_{0}}{\epsilon_{b}+J_{i}} .
$$

Let us estimate the threshold and ablation rate for the dental enamel. Dental enamel consists of approximately $15 \%$ water and $85 \%$ hydroxyapatite (HAP) of chemical composition $\mathrm{Ca}_{10}\left(\mathrm{PO}_{4}\right)_{6}(\mathrm{OH})_{2}\left(\mathrm{HPO}_{4}\right)_{0.15}\left(\mathrm{CO}_{3}\right) .{ }^{18}$ The average atomic number is $M \cong 21.8 \mathrm{amu}$, and the atomic number density $n_{a}=7.8 \times 10^{22}$ atoms $/ \mathrm{cm}^{3}$. We assume that the average binding energy $\epsilon_{b}$ is $5 \mathrm{eV}$ and that the average ionization potential for this complex material is $12 \mathrm{eV}$ per atom like for good dielectrics. The ablation threshold, determined using Eq. (3) is $0.95 \mathrm{~J} / \mathrm{cm}^{2}$, which is in reasonable agreement with the experimentally determined value of $2.2 \mathrm{~J} / \mathrm{cm}^{2}$. We should note here that with the help of the scaling laws, Eqs. (2)-(4), it is more practical to retrieve the unknown parameters for complex materials, such as the sum of the binding energy and ionization potential, from the experimentally measured threshold and ablation rate. Taking $A I_{0} \sim 10^{14} \mathrm{~W} / \mathrm{cm}^{2}$ as a characteristic parameter for ultrashort mode, one obtains from Eq. (4) a typical ablation rate of $\sim 4 \times 10^{31} \mathrm{~cm}^{-2} \mathrm{~s}^{-1}$. This ablation rate is almost four orders of magnitude higher than that in the thermal regime of evaporation with nanosecond laser pulses, and about 100 times higher than with $60 \mathrm{ps}$ pulses. $^{16}$

The ablated depth per pulse can be obtained by multiplying the ablation rate by the ablated area and the pulse duration, and taking into account the atomic number density of the enamel. The resultant value of $0.7 \mu \mathrm{m}$ per pulse fits well with the experimentally measured ablated depth (Fig. 3) in the laser fluence range $(1-10) \times F_{\text {th }}$. However, for higher laser intensities and for multiple laser shots per site this prediction should be used with caution. As the laser intensity increases, so does the energy loss for heating the ablated ions in the plasma layer, and for the next stages in the ionization. Thus, the pathway toward an increase of the total evaporation rate per second lies in the increase of the laser repetition rate, but not in an increase of laser intensity.

The formulas presented above do not contain any fitting parameters and agree well with experimental data available. In the new regime of electrostatic ablation with subpicosecond laser pulses the threshold fluence is almost independent of the pulse duration in the subpicosecond range, and the material evaporation rate is much higher than that in the long pulse interaction regime.

We would like to note that due to the transient character of all the material characteristics used for the fs laser ablation estimates one cannot expect a perfect quantitative fit to experimental data with the simple theoretical analysis pre- 
sented in this article. For example, the thermodiffusivity changes almost two times during the ablation process. Moreover, the electrostatic regime of ablation applies only at the time period during the subpicosecond pulse and under the condition that the energetic electrons on the target surface gain energy in the laser field higher than the Fermi energy, which is approximately the sum of the binding energy and ionization potential: ${ }^{9}$

$$
T_{e}>\left(\epsilon_{b}+J_{i}\right) \text {. }
$$

When the electron temperature is lower than this sum, the electrons do not have sufficient energy to pull ions out of the target. Thus, there is a mixture of electrostatic and thermal regimes during the course of ablation, while the thermal mode is the only mechanism after the pulse. Nevertheless, the electrostatic regime of ablation is the initial mechanism of ion removal and thus it determines the ablation threshold. The estimates of ablation threshold presented based on the electrostatic mechanism of ablation offer a very useful and simple way of determining the laser ablation threshold, within a factor of 2 according to our analysis, especially for complex dielectric materials such as dental enamel.

\section{CONCLUSIONS}

We have measured the threshhold energy fluence for subpicosecond laser ablation of dental enamel to be $2.2 \pm 0.1$ $\mathrm{J} / \mathrm{cm}^{2}$ for $800 \mathrm{~nm}$ wavelength light whereas it was $1.0 \pm 0.2$ $\mathrm{J} / \mathrm{cm}^{2}$ for $400 \mathrm{~nm}$ laser output. This suggests that the subpicosecond laser ablation source should be chosen by considering the wavelength of operation, because the rise in intrapulpal temperature may also be affected by this parameter.

The demonstrated linear dependence of the ablated mass on the laser intensity permits the material removal rate to be easily predicted. The ablation rate of material removal with $150 \mathrm{fs}$ pulses is $\sim 1 \mu \mathrm{m} /$ pulse at $20 \mathrm{~J} / \mathrm{cm}^{2}$ or, taking into account the focal spot area, $\sim 2 \times 10^{-3} \mathrm{~mm}^{3} / \mathrm{s}$, which is relatively high for laser ablation of dental tissue. Mechanical drill tissue removal rates are of the order of $0.5-1 \mathrm{~mm}^{3} / \mathrm{s}$, depending on the cutting tool chosen. ${ }^{19}$ To obtain comparable tissue removal rates with subpicosecond laser ablation with the same pulse duration, laser fluence, and focal spot size would require pulse repetition rates of the order of 200$500 \mathrm{kHz}$. However both carious dental tissue and dentine are softer than healthy enamel, so these would be expected to ablate up to 10 times more readily. ${ }^{11,20}$ Furthermore, the potential benefits of noncontact, painless laser treatment may provide clinical motivation for subpicosecond laser ablation of dental tissue at lower pulse repetition rates. No collateral thermal damage is observed in the prepared teeth, and the intrapulpal temperature is maintained below $5.5^{\circ} \mathrm{C}$ with the use of air cooling. Considering the electrostatic ablation mechanism, we do not expect a significant temperature in- crease, however, the laser pulse contrast ratio, with a low power nanosecond pedestal on the pulse, and the nonideal Gaussian pulse profile may contribute to the heating observed. A straightforward way by which to increase the laser contrast is to convert the laser radiation into the second harmonic. The improved contrast ratio together with the reduced threshold fluence demonstrated in this article are clear indications in favor of using the second harmonic of fundamental frequency for subpicosecond laser ablation.

Subpicosecond laser pulse ablation offers the potential to avoid or minimize the detrimental side effects of the dental drill to allow painless removal of hard tissue, and will greatly enhance the efficacy of clinical procedures and have broad applications to other types of laser surgery.

\section{ACKNOWLEDGMENTS}

The authors would like to acknowledge fruitful discussions and exchange of ideas with A. Kasenbacher, J. Serbin, and B. N. Chichkov. Financial support from the Australian Dental Research Foundation is greatly appreciated.

${ }^{1}$ J. Neev, L. B. Da Silva, M. D. Feit, M. D. Perry, A. M. Rubenchik, and B. C. Stuart, IEEE J. Sel. Top. Quantum Electron. 2, 790 (1996).

${ }^{2}$ A. A. Oraevsky, L. B. Da Silva, A. M. Rubenchik, M. D. Feit, M. E. Glinsky, M. D. Perry, B. M. Mammini, W. Small IV, and B. C. Stuart, IEEE J. Sel. Top. Quantum Electron. 2, 801 (1996).

${ }^{3}$ B.-M. Kim, M. D. Feit, A. M. Rubenchik, B. M. Mammini, and L. B. Da Silva, Appl. Surf. Sci. 127-129, 857 (1998).

${ }^{4}$ F. H. Loesel, M. H. Niemz, J. F. Bille, and T. Juhasz, IEEE J. Quantum Electron. 32, 1717 (1996).

${ }^{5}$ C. Momma, S. Nolte, B. N. Chichkov, F. v. Alvensleben, and A. Tunnermann, Appl. Surf. Sci. 109/110, 15 (1997).

${ }^{6}$ D. Du, X. Liu, J. Squier, G. Korn, and G. Mourou, Appl. Phys. Lett. 64, 3071 (1994).

${ }^{7}$ M. Lenzner, J. Krüger, W. Kautek, and F. Krausz, Appl. Phys. A: Mater. Sci. Process. 69, 465 (1999).

${ }^{8}$ M. D. Perry, B. C. Stuart, P. S. Banks, M. D. Feit, V. Yanovsky, and A. M. Rubenchik, J. Appl. Phys. 85, 6803 (1999).

${ }^{9}$ E. G. Gamaly, A. V. Rode, V. T. Tikhonchuk, and B. Luther-Davies, Phys. Plasmas 9, 949 (2002).

${ }^{10}$ E. G. Gamaly, A. V. Rode, V. T. Tikhonchuk, and B. Luther-Davies, Conference Digest CLEO-Europe, Nice, 2000.

${ }^{11}$ J. Serbin, T. Bauer, C. Fallnich, A. Kasenbacher, and W. H. Arnold, Appl. Surf. Sci. (to be published).

${ }^{12}$ L. Zach and G. Cohen, Oral Surg., Oral Med., Oral Pathol. 19, 515 (1965).

${ }^{13}$ B. Luther-Davies, E. G. Gamaly, Y. Wang, A. V. Rode, and V. T. Tichonchuk, Sov. J. Quantum Electron. 22, 289 (1992).

${ }^{14}$ D. Bäuerle, Laser Processing and Chemistry, 3rd ed. (Springer, Berlin, 2000), Chap. 13, and references therein.

${ }^{15}$ Yu. V. Afanasiev, B. N. Chichkov, N. N. Demchenko, V. A. Isakov, and I. N. Zavestovskaya, J. Russ. Laser Res. 20, 489 (1999).

${ }^{16}$ E. G. Gamaly, A. V. Rode, and B. Luther-Davies, J. Appl. Phys. 85, 4213 (1999).

${ }^{17}$ A. V. Rode, B. Luther-Davies, and E. G. Gamaly, J. Appl. Phys. 85, 4222 (1999).

${ }^{18}$ M. Ostertag, J. T. McKinley, L. Reinisch, D. M. Harris, and N. H. Tolk, Lasers Surg. Med. 21, 384 (1997).

${ }^{19}$ G. B. Altshuler, A. V. Belikov, and Y. A. Sinelnik, Lasers Surg. Med. 28, 435 (2001).

${ }^{20}$ M. H. Niemz, J. Dent. Res. 74, 1194 (1995). 